\title{
Caracterización de Accesiones de Chile (Capsicum spp.) ${ }^{1}$
}

\author{
Nora C. Martín 2 y William G. González ${ }^{3}$
}

\section{COMPENDIO}

Se trabajó con una población de 59 accesiones pertenecientes a una colección de Chile (Capsicum spp.) del Banco de Germoplasma del Centro Agronómico Tropical de Investigación y Enseñanza (CATIE) y dos del Programa de Recursos Fitogenéticos de la Universidad de Costa Rica (UCR). El trabajo de campo se realizó en la Estación Experimental Fabio Baudrit (UCR) en Alajuela, en el período comprendido de enero a septiembre de 1988. Se utilizó una metodología de semillero y luego el trasplante al campo definitivo.

Se caracterizaron las accesiones y con los datos recavados se logró asociar algunas características cualitativas importantes, mediante la utilización de correlaciones simples; también se relacionaron algunas características cuantitativas con cualitativas y entre características cualitativas, para las que se utilizó la ecuación propuesta por Engels.

Se logró determinar como el grosor de la pulpa correlacionó positivamente con el peso fresco (0.75) con el número de semillas (0.59) y con la longitud del fruto (0.53) y débilmente de manera negativa con la pungencia (-0.42) y con los días a floración $(-0.36)$. El diámetro del fruto correlacionó positivamente con: el número de semillas por fruto (0.62) grosor de la pulpa (0.72) y ésta a su vez con el número de semillas por fruto $(0.51)$.

Con las relaciones propuestas por Engels se asociaron 21 pares de características entre sí, como por ejemplo, la mayoría de los frutos más pesados se asociaron a posiciones de éstos péndulos con plantas de desarrollo precoz y flor de color blanco.

Palabras claves adicionales: caracterización, recursos fitogenéticos.

\section{INTRODUCCION}

El chile (Capsicum spp.) se utiliza de di versas foonas, fresco, seco, como especia en polvo, como colorante natural, como agente antioxidante, como bactericida y fungicida, como medicamento en la industria farmacéutica, en la industria cosmetológica, en industria de embutidos y carnes enlatadas. Por su potencialidad de comercialización, se ha identificado como uno de los cultivos prioritarios para investigación y desarrollo por parte del Programa de Recursos Fitogenéticos de la Estación Experimental Fabio Baudrit (EEFB), Universidad de Costa Rica, y de la Coalición Costarricense de Iniciativas de Desarrollo (CINDE). Por otra parte, el Centro Agronómico Tropical de Investigación y Enseñanza (CATIE), cuenta con la colección mundial de Capsicum, la cual presenta un alto porcentaje de sus introducciones sin caracterizar. El

\begin{abstract}
4
A population of 59 accessions of pepper (Capsicum spp) from the Germplasm Bank ofthe Centro Agronomico Tropical de Investigacion y Ensenanza (CATIE) and two populations from the Genetic Resources Program of the Vniversity of Costa Rica (UCR) were evaluated. Field experiments were conducted at the Fabio Baudrit UCR Experiment Station in Alajuela. Trials were conducted during the months of January through September of 1989. Seedlings were established under greenhouse conditions and later transplanted in the field.
\end{abstract}

The populations were characterized with data obtained from field experiments. Various quantitative and qualitative characteristics were related to one another using simple linear correlations. Qualitative characteristics were related using the Engel's equation.

Pulp thickness was positively correlated with fruit fresh weight $(\mathrm{r}=0.75)$, seed number $(\mathrm{r}=0.59)$ and fruit length $(\mathrm{r}=0.53)$; and negatively correlated with pungency $(\mathrm{r}=-0.42)$ and days to flower $(\mathrm{r}=-0.36)$. Fruit diameter was posit'ively correlated with seed number $(r=0.62)$ and pulp thickness $(\mathrm{r}=0.72)$. Pulp thickness also was positively correlated with seed number $(\mathrm{r}=0.51)$.

Using the relationships proposed by Engels, 21 pairs of characteristics were related. For example, the majority of the heaviest fruits were positively correlated with early maturing white flower plants.

conocimiento de las características de los materiales de esta colección es de invaluable importancia para el desarrollo de futuros trabajos de mejoramiento, o para la utilización de los mismos en programas de producción.

En base a los anterior se llevó a cabo el presente trabajo el cual tuvo como objetivos:

${ }^{1}$ Trabajo presentado en la XXXVI Reunión Anual del PCCMCA. San Salvador, marzo 1990.

2 Ing. Agrónomo. Datos parciales de tesis de grado. Facultad de Agronomía. Universidad de Costa Rica. 1990.

3 Ing. Agr. Programa Recursos Fitogenéticos, Estación Experimental Fabio Baudrit, Universidad de Costa Rica. EEFB/UCR-Apdo. 183 4050, Alajuela, Costa Rica.

${ }^{4} \mathrm{El}$ abstract es traducción del compendio.

Publicado en Agronomía Mesoamericana, Vol. 2 (1991). 
- La caracterización de 59 introducciones de Capsicum ssp. del Banco de Germoplasma del CATIE.

- Determinar el grado de correlación entre algunas características cualitativas y cuantitativas.

\section{REVISIÓN DE LITERATURA}

El chile es considerado una de las primeras plantas cultivadas de Mesoamérica y la continuidad de su uso se confirma desde 7000 y 5000 años A.C (Long-Solis, 1986).

El género Capsicum es un género muy homogéneo, pero aún los botánicos no han llegado a un concenso con la división de las especies. Algunos mencionan 90 y otros 12. Actualmente se reconocen 5 especies cultivadas: C. Baccatum C. Pubescens, C. Frutescens. C. Chinense y C. annuum (IBPGR, 1983).

La caracterización morfológica de los materiales fitogenéticos es una actividad que permite la selección de los materiales más promisorios para su posterior utilización en programas de mejoramiento. De acuerdo al Consejo Internacional de Recursos Fitogenéticos (IBPGR, 1980), la caracterización consiste en registrar todas aquellas caracterizaciones que son altamente heredables, que pueden verse fácilmente y que son expresadas en todos los ambientes. Según Engels (1979), las descripciones morfológicas, agronómicas, fisiológicas, etc. de una caracterización deberían ser acompañadas de información relacionada con prácticas culturales, condiciones ambientales, etc. y además, las colecciones que se describen deben crecer bajo condiciones uniformes para asegurar de esta manera que las diferencias registradas sean típicas de los materiales bajo esas circunstancias.

En documentación genética cada término descriptivo se llama un descriptor, tal puede ser el "número de introducción", "color del fruto", etc. El valor o grado de un descriptor se llama estado del descriptor. Si el descriptor se refiere a una caracterización cuantitativa, como la longitud del fruto o rendimiento, el estado del descriptor se expresa en la unidad de medida usada $(\mathrm{cm}, \mathrm{mm}, \mathrm{g})$, o la medida puede codificarse para facilitar el almacenamiento de datos. Cuando el descriptor se refiere a una caracterización cualitativa, como color o forma, los estados del descriptor se pueden basar en una tabla de colores o definiciones geométricas, respectivamente. Estos términos pueden codificarse si fuera conveniente. El estado del descriptor de una característica particular se registra con " 0 " cuando está ausente o no puede medirse; por otra parte, se expresa como "+", cuando se encuentra presente pero no se le da grado (Programa de Recursos Fitogenéticos CATIE/GTZ, 1979).

Trabajos efectuados apoyan la importancia del género Capsicumen el mundo y han proporcionado aportes significativos en el conocimiento del género. Así, se ha determinado que las características de color y forma del fruto son de poco valor taxonómico debido a la variación que existe dentro de la misma especie (Smith y Heiser, 1951).

Según Pickersgill (1968) la posición del fruto es una característica distintiva entre materiales silvestres y domesticados, ya que las poblaciones silvestres presentan la posición del fruto erecto, mientras que en los materiales cultivados con mayor grado de domesticación la posición del fruto es colgante.

El color de la corola de las flores, es una caracterí tica, que usada con otras características descriminantes, se emplea para delimitar especies. Así, en estado fresco la flor de la especie $C$. annuum tiene una coloración blanca lechosa, la flor de la especie $C$. frutescens es de color blanco-verde y la flor de la especie $C$. chinense blanco-verde o blanco-mate. Las manchas en la corola es una característica propia de la especie $C$. baccatum y pueden ser amarillas, café o pardas. La especie $C$. pubescens tiene la corola de color morada. Además esta especie presenta frutos con semillas negras y rugosas (IBPGR, 1983; Smith y Heiser, 1951).

\section{MATERIALES Y MÉTODOS}

El trabajo se realizó en la Estación Experimental Fabio Baudrit, de la Universidad de Costa Rica, localizada en San José de Alajuela, a una altitud de 840 m. La región es

Cuadro 1 Datos climáticos correspondientes al período de caracterización de las 57 introducciones de chile (Capsicum spp.) del CATIE EEFBM. Alajuela, Costa Rica.

\begin{tabular}{lcc}
\hline Mes & Temperatura $\left({ }^{\circ} \mathrm{C}\right)$ & Lluvia $(\mathrm{mm})$ \\
\hline Febrero & 23.7 & 0.0 \\
Marzo & 23.6 & 57.0 \\
Abril & 23.8 & 82.8 \\
Mayo & 22.8 & 323.7 \\
Junio & 21.4 & 241.4 \\
Julio & 21.7 & 147.8 \\
Agosto & 21.0 & 301.0 \\
Septiembre & 20.8 & 568.2 \\
Octubre & 20.8 & 449.3 \\
\hline
\end{tabular}

Humedad relativa: $89.3 \%$ Brillo Solar: 6.8

Cuadro 2 Datos químicos del suelo $(0-20 \mathrm{~cm})$ donde fueron trasplantadas las accesiones de chile (Capsicum spp.) escogidas para caracterización. Lote \#9 EEFBM, Alajuela, Costa Rica.

\begin{tabular}{ccc}
\hline Elemento & meg/100 g de suelo & ppm \\
\hline $\mathrm{K}$ & 1.07 & \\
$\mathrm{Ca}$ & 8.40 & \\
$\mathrm{Mg}$ & 2.72 & \\
$\mathrm{Al}$ & 0.20 & \\
$\mathrm{Mn}$ & & 19 \\
$\mathrm{Zn}$ & & 1 \\
$\mathrm{Cu}$ & & 18 \\
$\mathrm{P}$ & & 15 \\
$\mathrm{Fe}$ & & 263 \\
\hline
\end{tabular}

\% materia orgánica $=8.11: \mathrm{pH}: \mathrm{H}_{2} \mathrm{O}=5.6 ; \mathrm{HCl}=4.7$ 
Cuadro 3 Datos de pasaporte de las accesiones de chile (Capsicum spp.) escogidas para caracterizar en la Estación Experimental Fabio Baudrit M. Alajuela, Costa Rica, 1988.

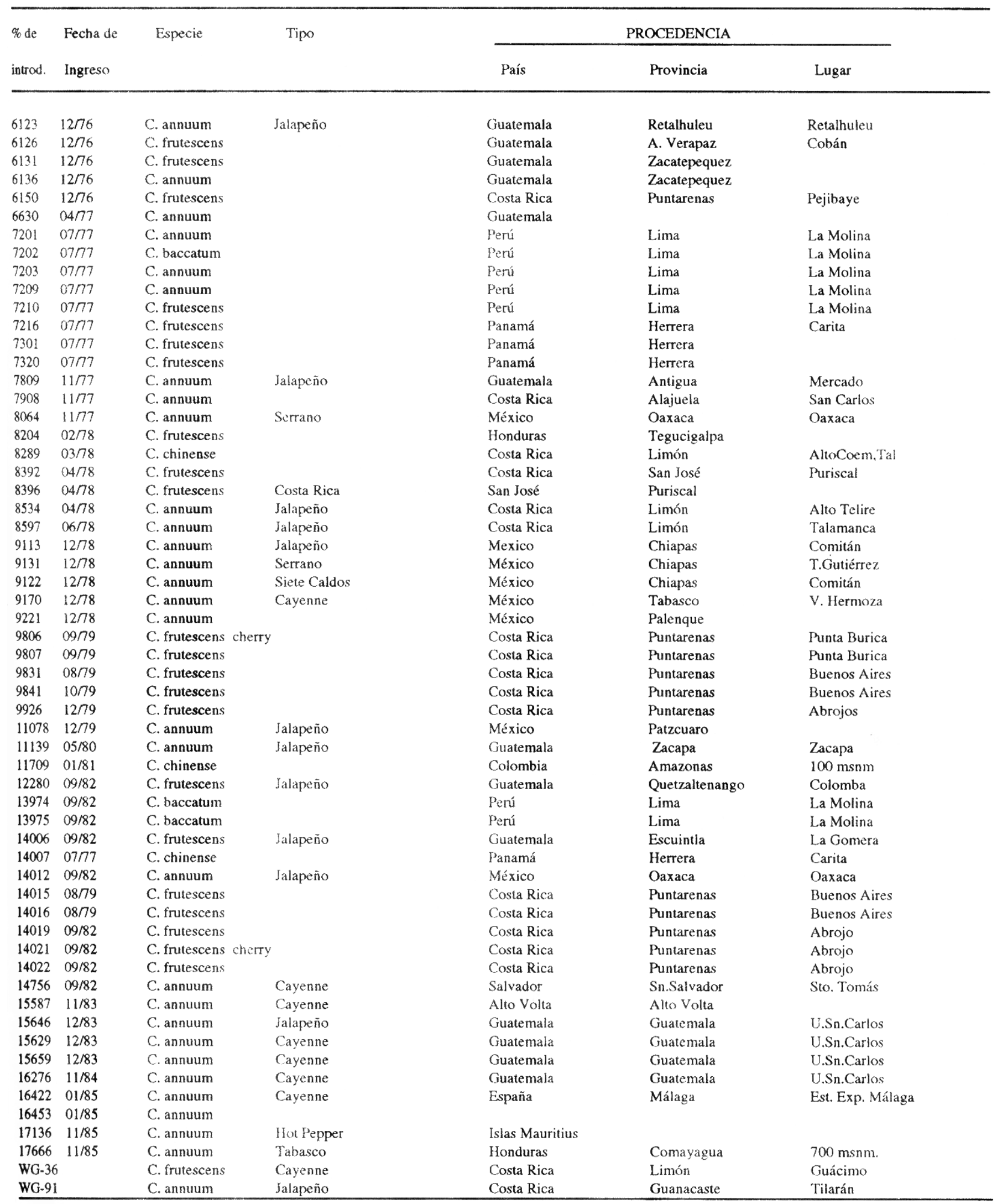

Fuente: Datos tomados de los libros de registro del Banco de Germoplasma del CATIE. 
clasificada ccológicamente como bosque húmedo premontano, con una precipitación media anual de 1930 $\mathrm{mm}$ distribuida de mayo a noviembre. Con una temperatura media de $22.4{ }^{\circ} \mathrm{C}$ con máximas de $2.8^{\circ} \mathrm{C}$ y mínimas de 18.2 ${ }^{\circ} \mathrm{C}$. Los datos climatológicos correspondientes al período de evaluación y las características químicas del suelo donde se transplantó el ensayo se resumen en los Cuadros 1 y 2.

Los trabajos de caracterización se efectuaron con 59 accesiones de chile (Capsicum spp.) provenientes del Banco de gemloplasma del CATIE. En el Cuadro 3 se indica la especie reportarda en los datos de pasaporte y procedencia de cada una de las especies escogidas.

Las plantas para la caracterización se obtuvieron de semilleros hechos bajo condiciones de invernadero, para lo cual se emplearon vasos plásticos de $275 \mathrm{ml}$ con un substrato de una mezcla en las siguientes proporciones: 5 partes de suelo, 3 partes de granza de arroz, 1 parte de gallinaza. El substrato fue tratado previamente con bromuro de metilo. Se sembraron 4 semillas por vaso y luego se dejó una planta. El transplante del ensayo se efectuó alos 39 días de la siembra cuando las plantitas presentaron de 4 a 6 hojas bien desarrolladas. La preparación de 1 terreno de siembra fiJe mecanizada y consistió de una arada, una rastreada y construcción de lomillos a un metro. Se sembraron 9 plantas por introducción en parcelas de 4.5 metros cuadrados, las cuales consistieron de 3 lomillos separados a $1.0 \mathrm{~m}$ y 3 plantas por lomillo separadas a $0.5 \mathrm{~m}$.

El mantenimiento de las plantas se realizó de acuerdo a las recomendaciones del Programa de Vegetales Procesados del CINDE-División Agrícola.

La selección de las variables para la caracterización de jas introducciones se hizo de acuerdo con la lista de descriptores para Capsicum spp., preparada por el Consejo Internacional de Recursos Fitogenéticos (IBPGR, 1983).

La caracterización se realizó de acuerdo con la escala de 0 a 9 propuesta por Astorga y Seidewitz (1983) (Cuadro 4). Las características cualitativas doble estado se registraron de la siguiente forma: 0 = falta de expresión fenotípica; $+=$ presencia de expresión fenotípica.

El análisis de la información se efectuó tomando en consideración el comportamiento exhibido por la población, debido a que las introducciones utilizadas en este trabajo corresponden a las colectas de poblaciones de plantas y no a líneas dentro de la población.

Para las características cuantitativas se utilizaron los datos originales y se relacionaron con correlaciones simples como mejor manera para determinar en que medida describe o explica, de una forma adecuada, la relación entre variables (Spiegel, 1982).

Para el análisis de relación entre características cuantitativas y cualitativas, así como de las características cualitativas entre sí, se utilizó el método propuesto por Engels (1978). Tal método consiste en relacionar por pares, las características.

La población fue analizada con índices de variación como promedios, valores mínimos, valores máximos y desviación estándar.

\section{RESULTADOS Y DISCUSIÓN}

Distribución de frecuencias y porcentajes de las características cualitativas estudiadas.

En el Cuadro 5, se muestra la distribución de frecuencias y porcentajes de las características estudiadas. ton respecto a la variable hábito de crecimiento de las plantas, se obtuvo que las 59 introducciones solamente la 11709 y la 7320 presentaron hábito de crecimiento rastrero, lo que equivale a un $3.39 \%$ del total, el restante $96.61 \%$ presentó hábitos de crecimiento compacto y erecto, el cual se prefiere pues facilita el manejo de planta, así como la aplicación de los agroquímicos y los frutos están menos expuestos al contagio por hongos del suelo.

Por otra parte, al ser compacta o erecta la planta tendrá mayor número de ramas principales que de acuerdo a Solanki y colaboradores (1986) correlaciona positivamente con el rendimiento.

Se encontró gran variabilidad en la característica pubescencia del tallo (Cuadro 5), donde el menor procentaje fue el de las introducciones glabras con un $15.25 \%$ y el

Cuadro 4 Condificación de grados de expresión de características, basada en la escala de 1 a 9.

\begin{tabular}{|c|c|c|c|c|c|c|c|c|c|}
\hline No.Ejemplos & 1 & 2 & 3 & 4 & 5 & 6 & 7 & 8 & 9 \\
\hline I & Muy bajo & Muy bajo a bajo & Bajo & Bajo a intermedio & Intermedio & Intermedio a alto & Alto & Alto a Muy alto & Muy alto \\
\hline II & Muy bajo & --- & Bajo & --- & Intermedio & --. & Alto & ... & Muy alto \\
\hline III & -.. & Muy bajo a bajo & -- & Bajo a intermedio & --- & Intermedio a alto & -- & Alto a muy alto & ... \\
\hline IV & $\ldots$ & 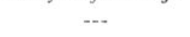 & Bajo & $-\ldots$ & Intermedio & $-\cdots$ & Alto & -.- & $\ldots$ \\
\hline $\mathrm{V}$ & $\ldots$ & $\ldots$ & Bajo & $\ldots$ & --. & $\ldots$ & Alto & $\ldots$ & $\ldots$ \\
\hline VI & Muy bajo &.-- & -.. & $\ldots$ & Intermedio &..- & ... & $\ldots$ & Muy alto \\
\hline VII & Muy bajo & -- & -.- & -.- & Intermedio & -- & -.- & $\ldots$ & Muy alto \\
\hline
\end{tabular}

Fuente: Astorga y Seidewitz L. (1983). 
Cuadro 5 Frecuencia y porcentaje de las características cualitativas estudiadas a las introducciones de chile (Capsicum spp.) caracterizadas. Est. Exp. Fabio Baudrit Moreno. Alajuela, Costa Rica.,1988.

\begin{tabular}{|c|c|c|c|c|}
\hline Características & Código & o Clase & Frecuencia $\mathrm{F}$ & Porcentaje \\
\hline \multirow[t]{3}{*}{ Hábito de crecimiento } & 3 & postrado & 2 & 3.39 \\
\hline & 5 & compacto & 35 & 59.32 \\
\hline & 7 & erécto & 22 & 37.29 \\
\hline \multirow[t]{4}{*}{ Pubescencia del tallo } & 0 & glabro & 9 & 15.25 \\
\hline & 3 & ralo & 21 & 35.59 \\
\hline & 5 & intermedio & 13 & 22.04 \\
\hline & 7 & abundante & 16 & 27.12 \\
\hline \multirow[t]{4}{*}{ Pubescencia de la hoja } & 0 & glabro & 11 & 18.64 \\
\hline & 3 & ralo & 27 & 45.76 \\
\hline & 5 & intermedio & 14 & 23.73 \\
\hline & 7 & abundante & 7 & 11.78 \\
\hline \multirow{2}{*}{$\begin{array}{l}\text { Número de pedicelos } \\
\text { por áxila }\end{array}$} & 1 & & 49 & 83.05 \\
\hline & 2 & & 10 & 16.95 \\
\hline \multirow{3}{*}{$\begin{array}{l}\text { Posición del pedicelo } \\
\text { en la antesis }\end{array}$} & 3 & péndulo & 26 & 44.07 \\
\hline & 5 & intermedio & 13 & 22.04 \\
\hline & 7 & erécto & 20 & 33.89 \\
\hline \multirow[t]{2}{*}{ Color de la corola } & 1 & blanco & 3 & 55.93 \\
\hline & 2 & blanco-verde & 26 & 44.07 \\
\hline \multirow{3}{*}{$\begin{array}{l}\text { Forma del margen del } \\
\text { cáliz }\end{array}$} & 3 & liso & 5 & 8.48 \\
\hline & 5 & intermedio & 34 & 57.63 \\
\hline & 7 & dentado & 20 & 33.89 \\
\hline \multirow{2}{*}{$\begin{array}{l}\text { Constricción anular eì } \\
\text { unión cáliz-pendúnculo }\end{array}$} & 0 & ausente & 46 & 77.96 \\
\hline & 1 & presenti & 13 & 22.04 \\
\hline \multirow[t]{3}{*}{ Posición del fruto } & 3 & péndulo & 33 & 55.93 \\
\hline & 5 & intermedio & 9 & 15.25 \\
\hline & 7 & erécto & 17 & 28.82 \\
\hline \multirow[t]{3}{*}{ Color del fruto inmaduro } & 1 & verde & 38 & 64.41 \\
\hline & 2 & verde claro & 13 & 22.03 \\
\hline & 3 & amarillo & 8 & 13.56 \\
\hline \multirow[t]{6}{*}{ Forma del fruto } & 1 & elongado & 41 & 69.49 \\
\hline & 2 & oblongo & 2 & 3.39 \\
\hline & 3 & redondeado & 1 & 1.69 \\
\hline & 4 & cónico & 6 & 10.18 \\
\hline & 5 & campanulado & 2 & 3.39 \\
\hline & 6 & acampanado & 1 & 1.86 \\
\hline \multirow[t]{2}{*}{ Persistencia del fruto } & 0 & caduco & 16 & 27.12 \\
\hline & + & persistente & 43 & 72.88 \\
\hline \multirow[t]{3}{*}{ Mancha en la corola } & 0 & ausente & 53 & 89.83 \\
\hline & 1 & blanco & 0 & 0 \\
\hline & 2 & amarillo & 6 & 10.17 \\
\hline \multirow[t]{5}{*}{ Color de la antera } & 1 & amarillo & 8 & 13.56 \\
\hline & 2 & azul pálido & 28 & $\$ 7.46$ \\
\hline & 3 & azul & 21 & 3.5 .59 \\
\hline & 4 & púrpura & 2 & 3.39 \\
\hline & 5 & otro & 0 & 0 \\
\hline \multirow[t]{2}{*}{ Posición del estigma } & 3 & incluido & 1 & 1.69 \\
\hline & 5 & mismo nivel & 9 & 15.25 \\
\hline
\end{tabular}

\begin{tabular}{|c|c|c|c|c|}
\hline & 7 & más alto & 49 & 83.06 \\
\hline \multirow[t]{2}{*}{ Cuello en base del fruto } & 0 & ausente & 52 & 88.14 \\
\hline & 1 & presente & 71 & 1.86 \\
\hline \multirow{5}{*}{$\begin{array}{l}\text { Forma del fruto en la } \\
\text { unión con el pedúnculo }\end{array}$} & 1 & agudo & 12 & 20.34 \\
\hline & 3 & obtuso & 15 & 25.42 \\
\hline & 5 & truncado & 22 & 37.29 \\
\hline & 7 & cordado & 10 & 16.95 \\
\hline & 9 & lobado & 0 & 0 \\
\hline \multirow{3}{*}{$\begin{array}{l}\text { Forma del fruto en el } \\
\text { extremo inferior }\end{array}$} & 3 & punteado & 40 & 67.79 \\
\hline & 5 & obtuso & 14 & 23.74 \\
\hline & 7 & hundido & 5 & 8.47 \\
\hline \multirow{4}{*}{$\begin{array}{l}\text { Corrugación del fruto } \\
\text { en sección transversal }\end{array}$} & 0 & liso & 18 & 30.51 \\
\hline & 3 & liger. corrugado & 22 & 37.29 \\
\hline & 5 & intermedio & 13 & 22.03 \\
\hline & 7 & muy corrugado & 6 & 10.17 \\
\hline
\end{tabular}

mayor de $35.59 \%$ para los materiales con pubescencia rala. Situación similar se presentó con respecto a la pubescencia de la hoja, donde la mayoría de los materiales presentaron pubescencia rala e intennedia.

Con relación al número de pedicelos por axila el $83.05 \%$ de los materiales mostró un solo pedicelo y el $16.95 \%$ presentó dos. Se deduce de los resultados, que esta característica puede ser utilizada para la separación entre especies, debido a la poca variabilidad que presenta (Smith y Heiger, 1951). Por el contrario la posición del pedicelo en la antesis presentó gran variabilidad (Cuadro 5).

Se presentaron solo dos colores de corola, el $55.93 \%$ de las introducciones fueron blancas y el $44.07 \%$ fueron blanco- verde. Por otra parte, las introducciones 7201, 7202 7203, 7209, 13974 y 13975 presentaron manchas amarillas en la corola, esta característica es propia de la especie $C$. baccatum (IBPGR, 1983).

El mayor porcentaje de los materiales (91.52\%) mostraron cáliz dentado o intennedio y solo el $8.48 \%$ lo tuvieron liso, característica asociada a plantas de la especie C. chinense (Long-Salís, 1986) y que fue mostrada por las introducciones $6126,8064,8204,8534$ y 9807 . Sin embargo, en los datos de pasaporte se reportan estos materiales como de las especies $C$. annuum y $C$. frutescens hecho que se puede corroborar el observar otras características como número de pedicelos y color de la corola. Por lo tanto, la fonna del margen del cálix no se puede considerar como una característica discriminante.

Las introducciones 7202, 7202, 7203, 7209, 11709, 13974, 13975 y 17136, presentaron anteras de color amarillo, que son propias de la especie $C$. baccatum, al igual que las manchas en la corola. Sin embargo, algunos de estos materiales se reportan en los datos de pasaporte como pertenecientes a otras especies, por lo que dichos datos deben ser revisados y corregidos.

Con respecto a la posición del estigma en relación a las anteras, 49 de las introducciones presentaron el estigma más 
alto que las anteras, 9 al mismo nivel y solo una más abajo dentro de las anteras, que correspondió a la introducción 7203. El chile es autógamo pero ocasionalmente ocurren cruces debido al viento o a los insectos y el mayor porcentaje de polinización cruzada ocurre en flores cuyos estigmas son más altos que el nivel de los estambres (IBPGR, 1983).

De las introducciones estudiadas eI55.93\% presentaron los frutos en posición péndula, $28.82 \%$ en posición erecta y el $15.25 \%$ en posición intermedia. En los materiales con mayor grado de domesticación los frutos tienden a ser péndulos, mientras que los materiales silvestres presentan la posición del fruto erecta. (Pickergill, 1969).

Tanto para la característica color del fruto inmaduro, como las formas de éste presentaron una gran variabilidad en la población analizada (Cuadro 5), lo que corrobora lo indicado por Smith y Heiser (1951), quienes sostienen que dichas características son de poco valor taxonómico debido a la variación que existe dentro de la misma especie.

De las introducciones analizadas 13 presentaron constricción anular en la unión del cáliz con el pedúnculo, para un porcentaje del $22.04 \%$. Esta característica es utilizada para distinguir a $C$. chinense que la presenta, de $C$. frutescens en la cual está ausente (IBPGR, 1983).

En la población caracterizada un $72.88 \%$ de las introducciones presentó frutos persistentes. Esta característica es importante, pues mide la capacidad de un material de mantener su cosecha o por el contrario de que una vez que se madura, se desprenda fácilmente. Para muchos de los usos que se le da al chile en la agroindustria, se requiere que estos se cosechen sin pedúnculo, por lo que es importante que estos se desprendan fácilmente a la hora de la cosecha.

Distribución de frecuencias y porcentaje de las características cuantitativas estudiadas

Según se puede observar en el Cuadro 6 el $15.25 \%$ de las introducciones fueron precoces (61-84 días) con respecto a los días a floración (días de la siembra hasta que el $50 \%$ de las plantas mostraban floración), el 71.19\% intermedias (85108 días) y el $13.56 \%$ tardías (109-132 días).

Los mismos porcentajes se observaron para los días a la primera cosecha donde las introducciones precoces tardaron de 76 a 96 días (a partir de la siembra del almácigo), las intermedias de 97 a 117 días y las tardías de 118 a 138 días.

La longitud del fruto es considerada como una de las características que más influye en el rendimiento (Achal et al. 1986). En la población estudiada la variabilidad fue alta (Cuadro 6), ubicándose el mayor porcentaje en plantas con longitud de frutos intermedios $(5.44-7.67 \mathrm{~cm})$. Un $18.65 \%$ mostrófrutos largos $(7.68-9.91 \mathrm{~cm})$ y solo el $5.08 \%$ frutos
Cuadro 6 Distribución de frecuencia y porcentajes de las características cuantitativas estudiadas a las introducciones de chile (Capsicum spp.) caracterizadas. Est. Exp. Fabio Baudrit Moreno, Alajuela, Costa Rica.

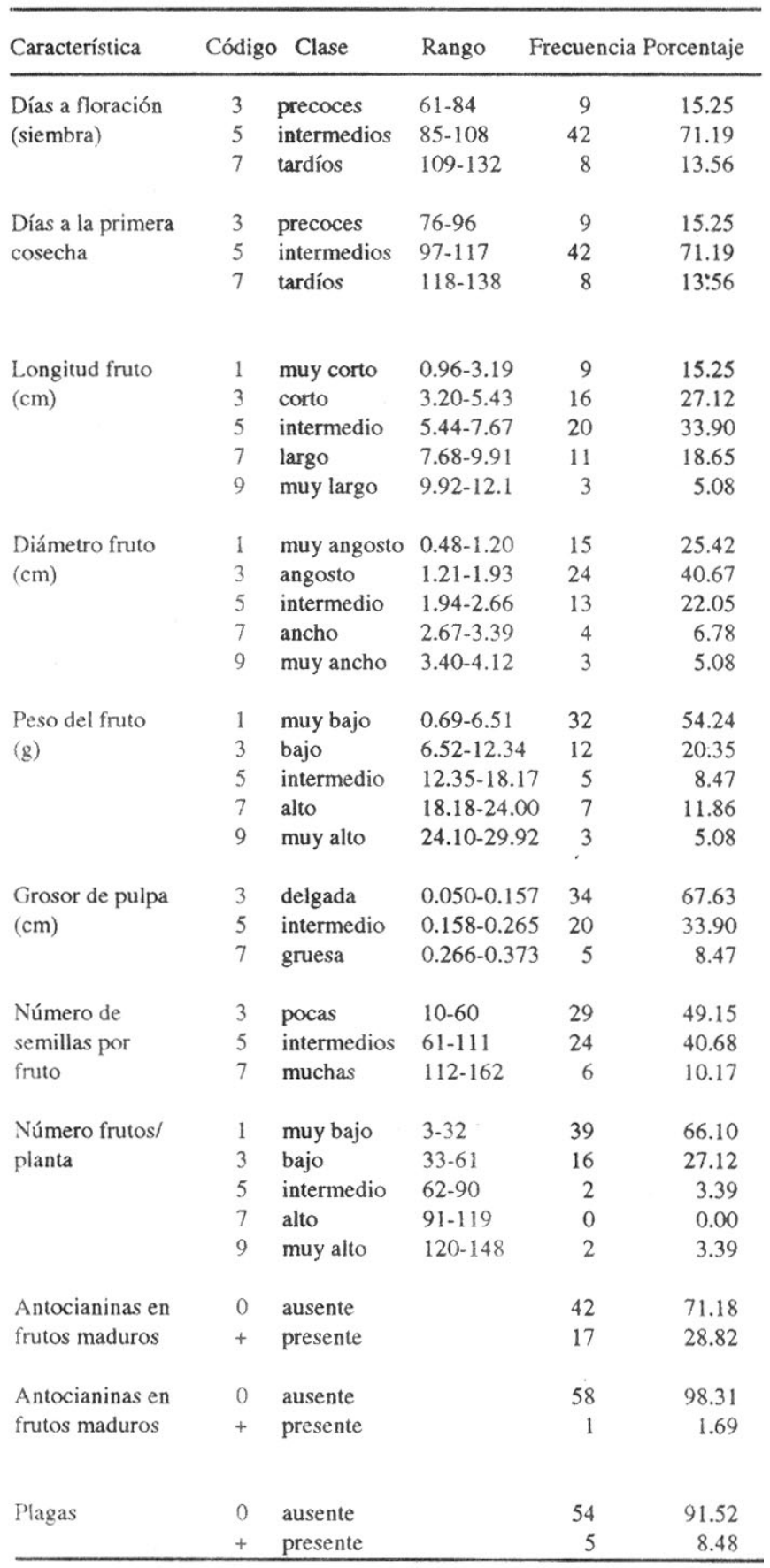

muy largos $(9.92-12.1 \mathrm{~cm})$. Situación similar ocurrió con el diámetro máximo del fruto, donde el mayor porcentaje se ubicó en plantas con frutos angostos $(1.21-193 \mathrm{~cm}$ ) y solamente 7 plantas mostraron frutos anchos o muy anchos. La característica ancho del fruto investigada entre otras por Achal et al (1986), resultó ser de alta capacidad heredable, por lo que recomiendan la selección de materiales tomando en cuenta el diámetro del fruto. 
La población estudiada mostró una mayor frecuencia de materiales con frutos de peso muy bajo o bajo (Cuadro 6). Las introducciones WG-36, 8392, 9122, 9170, 14006 y $15646 \mathrm{R}$ sentaron peso del fruto alto $(18,18-24.00 \mathrm{~g}$ por fruto) y solo las introducciones 7320, 14012 y 15646 mostraron frutos de peso muy alto (24,10-29.92 g). Esta característica es de gran importancia en el mejoramiento del chile, ya que éste se comercializa por peso.

Para la característica grosor de la pulpa el $91.53 \%$ presentó frutos de pulpa delgada e intermedia. Solo las introducciones WG-91, 13975, 14006, 14012, $15646 \mathrm{R}$ y 15646 mostraron frutos de pulpa gruesa, característica de importancia en frutos de chile jalapeño, o cuando los frutos se destinan a la confección de salsas.

Por otra parte, en los materiales analizados la mayor frecuencia fue para las introducciones con pocas semillas por fruto $\mathrm{y}$ frutos de contenido medio de semillas. Unicamente seis mostraron muchas semillas por fruto, correspondiendo a las introducciones 6136, 9122, 13974, 14006, 14012 y 16453. Para ciertos usos industriales del chile, la presencia de muchas semillas por fruto constituye una característica poco deseable, ya que afecta negativamente la calidad del producto final.

La mayoría de las introducciones presentaron muy bajo o bajo número de frutos por planta (Cuadro 6). Solamente las introducciones 14015 y 14016 presentaron un número intermedio (62-90 frutos/planta) y las introducciones 6126 y 6150 un número muy alto de frutos por planta (120-148 frutos/planta).

\section{Relaciones entre características cuantitativas}

Para las introducciones caracterizadas, se realizaron algunas correlaciones simples entre las características más importantes (Cuadro 7). De esta forma, se observó una correlación positiva entre las características peso del fruto contra longitud del fruto, número de semillas por fruto $\mathrm{y}$ grosor de la pulpa. Lo que significa que para los materiales analizados a mayor peso del fruto, este será más largo, más grueso de pulpa y con mayor número de semillas. Ello concuerda con lo reportado por González (1988) y Madrigal en an teriores trabajos de caracterización. Muchas agroindustrias utilizan esta correlación para sus estrategias de compra en el chile tipo jalapeño y/o serrano, al establecer rangos de pesos para las diferentes clases comerciales, ya que estos se asocian positivamente con la longitud del fruto.

Esta relación es de gran importancia al desarrollar programas de mejoramiento en chile picante ya que, a pesar de que este vegetal se compra por peso, los agroindustriales tienen diversas exigencias según sea el destino del producto final. Los industriales de chile seco necesitan frutos que no sean gruesos de pulpa para simplificar el proceso de deshidratación. Los procesadores deoleorresinas requieren frutos con pocas semillas, pues el contenido de grasas que
Cuadro 7 Coeficientes de correlación entre pares de características cuantitativas de 59 introducciones de chile (Capsicum spp.) Est. Ext. Fabio Baudrit M. Alajuela, Costa Rica, 1988

\begin{tabular}{|c|c|c|c|}
\hline Características & $\mathbf{r}$ & & \\
\hline \multirow[t]{8}{*}{ Peso del fruto contra: } & 0.53 & $* *$ & longitud \\
\hline & 0.41 & $* *$ & diámetro \\
\hline & -0.42 & & pungencia \\
\hline & 0.59 & $* *$ & número semillas \\
\hline & 0.75 & $* *$ & grosor pulpa \\
\hline & 0.46 & $*$ & peso seco 100 semillas \\
\hline & -0.36 & & días a floración \\
\hline & -0.30 & & días fructificación \\
\hline \multirow[t]{5}{*}{ Diámetro del fruto contra: } & 0.62 & & número semillas \\
\hline & 0.72 & & grosor pulpa \\
\hline & 0.49 & & peso seco 100 semillas \\
\hline & -0.25 & & días floración \\
\hline & -0.24 & & días fructificación \\
\hline \multirow[t]{5}{*}{ Semillas por fruto contra: } & 0.51 & $* *$ & grosor de pulpa \\
\hline & 0.59 & $* *$ & peso \\
\hline & -0.68 & $* *$ & pungencia \\
\hline & -0.47 & $* *$ & días floración \\
\hline & -0.46 & $* *$ & días fructificación \\
\hline
\end{tabular}

* Estadísticamente significativo a $\mathrm{P} £ 0.05$

** Estadisticamente significativo a P $£ 0.01$

poseen tienden a oxidarse en el proceso, desmejorando el color, y por ende la calidad de la oleorresina.

La relación entre peso del fruto y pungencia resultó debilmente negativa, por lo tanto frutos de mayor peso, son menos pungentes, Fernández (1984) encontró una correlación inversa entre el porcentaje de capsaicina y el tamaño del fruto. En su trabajo la alta pungencia en el fruto se relacionó con los chiles de tamaño pequeño y anaranjados.

Se obtuvo una correlación positiva entre el diámetro del fruto contra número de semillas por fruto y grosor de la pulpa. Según este resultado, entre más ancho es el fruto, presenta un mayor número de semillas y más gruesa es su pulpa.

La característica número de semilla por fruto, correlacionó positivamente con el grosor de la pulpa y peso del fruto (Cuadro 7), lo que corresponde a lo mencionado anteriormente de que frutos de pulpa gruesa son de mayor peso y mayor número de semillas.

Por otra parte, se dio una correlación inversa entre el número de semillas por fruto y la pungencia, o sea que los materiales que presentaron frutos con muchas semillas tienden a ser poco pungentes. Este resultado concuerda con lo obtenido por González (1985) en su trabajo de caracterización.

En cuanto a la relación de las características, número de semillas por fruto contra días a floración y días a fructificación se obtuvo una débil correlación negativa; anteriormente Madrigal (1988), obtuvo los mismos resultados. Por lo 
general, frutos que presentan muchas semillas provienen de plantas de desarrollo precoz.

\section{Relaciones entre características cuantitativas y cualitativas}

En el Cuadro 8 se presentan las principales relaciones entre características cuantitativas y cualitativas para lo cual se utilizó el método propuesto por Engels (1979) que consiste en relacionar por pares las características.

En las introducciones analizadas los frutos muy largos se asociaron a formas oblongas, de posición péndula y forma obtusa en la unión con el pedúnculo. Por lo contrario, los frutos muy cortos se asociaron con formas de redondeadas acónicas, posición erecta en la planta y forma truncada en la unión con el pedúnculo.

Tanto los frutos de diámetro ancho como los de diámetro angosto se relacionaron con posiciones intermedias a péndulas en la planta. Por otra parte, las plantas precoces en cuanto al número de días desde siembra a floración se asociaron a la posición péndula del pedicelo en la antesis, lo mismo que del fruto; contrariamente, las plantas tardías a floración se asociaron a las posiciones de intermedio a erecta del pedicelo en la antesis y a las mismas posiciones del fruto en la planta.

Las introducciones que presentaron frutos con un alto contenido de semillas se asociaron a las posiciones péndula o intermedia de los mismos; mientras que los frutos con bajo contenido de semillas mostraron posiciones de intermedia a erecta. Estas características se observan en las variedades comerciales de chile tipo tabasco ( $C$. frutescens) que se siembran en Costa Rica, las cuales se caracterizan por presentar frutos cortos, de forma cónica, posición erecta, bajo contenido de semillas, muy pungentes y plantas tardías en cuanto a días a floración y cosecha.

Con respecto a la pungencia, los frutos de picor bajo se asociaron a introducciones con chiles de diámetro angosto, posición intermedia a péndula en la planta y color blancoleche de la corola. Por el contrario los materiales con picor alto se asociaron a chiles con frutos muy angostos a angostos, de posición intermedia a erecta y colores blancoverde de la corola.

\section{Relaciones entre características cualitativas}

Al relacionar la posición del fruto con la forma de este (Cuadro 9), se encontró que en las introducciones caracterizadas, los frutos en posición péndula y erecta se asociaron con frutos oblongos y los de posición intermedia con frutos de forma oblonga y redondeados.

Cuando las flores presentaron anteras de color amarillo y azul pálido se relacionaron con corolas de color blanco, que pueden corresponder a las especies $C$. baccatum o C. annuит.
Cuadro 8 Algunas relaciones entre características cuantitativas y cualitativas de 59 introducciones de chile (Capsicum spp.) caracterizadas en la Est. Exp. Fabio Baudrit M. Alajuela, Costa Rica. 1988.

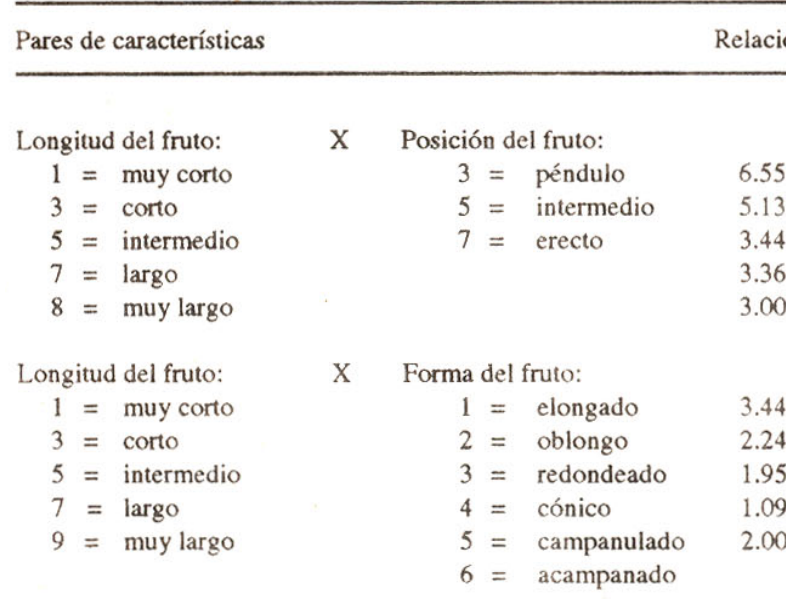

Longitud del fruto: $\quad \mathrm{X}$ Forma del fruto en la unión

$1=$ muy corto

$3=$ corto

$5=$ intermedio

$7=$ largo

$9=$ muy largo

Diámetro del fruto:

$1=$ muy angosto

3 = angosto

$5=$ intermedio

$7=$ ancho

$9=$ muy ancho

Posición del fruto:

3 = péndulo

$5=$ intermedio

7 = erecto

Días a floración

$3=$ precoces

$5=$ intermedias

$7=$ tardías

Número semillas:

3 = bajo

$5=$ intermedio

$7=$ alto

Picor:

$3=$ bajo

$5=$ intermedio

$7=$ alto

Picor:

3 = bajo

$5=$ intermedio

$7=$ alto

Picor:

3 = bajo

$5=$ intermedio

$7=$ alto con el pedúnculo:

$1=$ agudo

$3=$ obtuso

$5=$ truncado

$7=$ cordado

$9=$ lobado

5.22

3.12

3.84

4.82

3.00

X Posición del fruto:

$3=$ péndulo 5.71

$5=$ intermedió $\quad 4.22$

$7=$ erecto $\quad 3.62$

3.62
4.00

3.00

X Días a floración:

$3=$ precoces $\quad 4.57$

$5=$ intermedias $\quad 5.22$

$7=$ erecto $\quad 5.59$

X Posición del pedicelo en la antesis:

$3=$ péndulo $\quad 3.25$

$5=$ intermedio $\quad 4.87$

X Posición del fruto:

$3=$ péndulo $\quad 5.55$

$5=$ intermedio $\quad 3.25$

$7=$ erecto $\quad 4.00$

X Diámetro fruto:

$1=$ muy angosto 2.58

$3=$ angosto $\quad 2.55$

$5=$ intermedio $\quad 1.60$

X Posición del fruto:

$5=$ intermedio $\quad 4.11$

$7=$ erecto $\quad 5.60$

X Color de la corola:

$1=$ blanca
$7=$ erecto 6.11

$3=$ péndulo $\quad 3.33$

$\begin{array}{ll}4=\text { azul } & 1.77\end{array}$ 
Por otra parte, anteras azul o púrpura se asociaron con corolas verde, color típico de las flores de $C$. chinense o $C$. frutescens (IBPGR, 1983). La posición del fruto 'péndulo se ionó con flores de corola blanca, mientras que la posición ti se asoció con corolas blanco-verde.

Cuadro 9 Relaciones entre características de 59 accesiones de chile (Capsicum spp.), caracterizadas en la Est. Exp. Fabio Baudrit, Alajuela, Costa Rica, 1988.

\begin{tabular}{|c|c|c|c|}
\hline Pares de Características & & & Relación \\
\hline Posición del fruto: & $\mathrm{X}$ & Forma del fruto: & \\
\hline $3=$ péndulo & & $1=$ elongado & 2.00 \\
\hline $5=$ intermedio & & $2=$ oblongo & 2.66 \\
\hline $7=$ erecto & & $3=$ redondeado & 2.00 \\
\hline Posición del fruto: & $\mathrm{X}$ & Antocianinas en frutos inm & aduros: \\
\hline 3 = péndulo & & $+=$ presente & 0.22 \\
\hline $5=$ intermedio & & $0=$ ausente & 0.24 \\
\hline $7=$ erecto & & & 0.35 \\
\hline Color de antera: & $\mathbf{X}$ & Color de corola: & \\
\hline $1=$ amarillo & & $1=$ blanco & 1.13 \\
\hline $2=$ azul pálido & & $2=$ blanco-verde & 1.21 \\
\hline $3=$ azul & & $3=$ azul & 1.81 \\
\hline $4=$ púrpura & & $4=$ violeta & 2.00 \\
\hline Posición del fruto: & $\mathrm{X}$ & Color de la corola: & \\
\hline 3 = péndulo & & $1=$ blanco & 1.13 \\
\hline $5=$ intermedio & & $2=$ blanco-verde & 1.21 \\
\hline $7=$ erecto & & $3=\mathrm{azul}$ & 1.81 \\
\hline
\end{tabular}

\section{BIBLIOGRAFÍA}

ACHAL, S.; LAL, S.D.; PANT, C.e. 1986. Variability studies in chilli. Progressive Horticulture 18 (3-4) 270-272 p.

ASTROGA, C.; SEIDEWITZ, L. 1983. Documentación y comunicación en relación con los recursos fitogenéticos. Turrialba, Costa Rica, CA TIE. $168 \mathrm{p}$.
ENGELS, J. 1979. La documentación en Centros de Recursos Genéticos. Turrialba, Costa Rica. CA TIE. 12 p.

FERNANDEZ, S.S. 1984. Caracterizaciónqufmica y agronómica preliminar de 73 "tipos" de chile picante (Capsicum spp.) de la colección del CA TIE. . Tesis Lic. Tec. Alimentos. San José, Costa Rica. 70 p.

GONZALEZ,O.A. 1985. Caracterización de 10 introducciones de chile picante (Capsicum spp.) proveniente de varios países americanos, en Turrialba. Tesis Magister Scientiae, Turrialba, Costa Rica. Programa Universidad de Costa RicalCA TIE. 164 p.

INTERNATIONAL BOARD FOR PLANT GENETIC RESOURCES (IBPGR). 1980. Guidelines for developing descriptor listo Rome. $13 \mathrm{p}$.

INTERNATIONAL BOARD FOR PLANT GENETIC RESOURCES (IBPGR). 1983. Genetic Resourses of Capsicum. Roma, 49 p.

LONG-SOLIS, J. 1986. Capsicum y cultura. La historia del chilli. Fondo de Cultura Económico, México, 178 p.

MADRIGAL, L. A. 1988. Caracterización de 126 introducciones de chile picante (Capsicum spp.) del Banco de germoplasma del CATIE. Tesis de Ing. Agr., Sede Regional del Atlántico. Universidad de Costa Rica. 97 p

PICKERSGILL, B. 1969. The archeological record of chilli peppers. (Capsicum spp.) and the sequence of plant domestication in Perú. American Antiquity 34: 54-61 p.

PROGRAMA DE RECURSOS FITOGENETICOS CATIE/GTZ. 1979. Los recursos genéticos de las plantas cultivadas de América Central. San Juan de Tibás, Costa Rica, 32 p.

SMITH, P.; HEISER, jr. 1951. Taxonomic and genetic studies ofthe cultivated peppers, Capsicum annиum L. and Capsicum frutescens L. American Journal of Botany 38: 362-368 p.

SOLANKI, S.S.; SAXENA, P.K.; PANDEY I.C. 1986. Genotypic paths to fruit yiel in chilli (Capsicum annиит L.). Progressive Horticulture 18 (3-4): 227-229.

SPIEGEL, M.R. 1982. Estadística, Trad. por José Luis Gómez Espadas. McGraw-Hill Latinoamericana. S.A. Serie Schaum. 357 p. 